\title{
Representation of an equivalent circuit for capacitive wireless power transfer using a distributed-constant circuit
}

\author{
Yasumasa Naka ${ }^{1}$ and Masaya Tamura ${ }^{1, \text { a) }}$ \\ ${ }^{1}$ Department of Electrical and Electronic Information Engineering, \\ Toyohashi University of Technology, \\ 1-1 Hibarigaoka, Tempaku-cho, Toyohashi, Aichi 441-8580, Japan \\ a) tamura.masaya.yd@tut.jp
}

Abstract: Capacitive wireless power transfer is performed using large electrodes or using a medium having high relative permittivity such as water. The transfer efficiency demonstrates a pronounced change with frequency. A lumped-constant equivalent circuit with parasitic elements represents the change. However, a relationship between conditions for generating minimum/maximum values of the transfer efficiency and structural parameters of the electrodes in the coupler has not clarified. In this study, an equivalent circuit is proposed using a distributed-constant circuit. We converted the lumped-constant circuit into two open-ended directional couplers. From the result, we derived the conditions for generating the minimum and maximum values of the voltage input/output ratio. The efficiencies calculated by the distributed-constant circuit and electromagnetic simulation were in good agreement.

Keywords: wireless power transfer, capacitive wireless power transfer, directional coupler, equivalent circuit, maximum available efficiency

Classification: Energy in Electronics Communications

\section{References}

[1] T.M. Mostafa, D. Bui, A. Muharam, A.P. Hu, and R. Hattori, "Load effect analysis and maximum power transfer tracking of CPT system," IEEE Trans. Circuits Syst. I, Reg. Papers (Early Access), March 2020. DOI: 10.1109/TCSI.2020.2981195

[2] H. Zhang, F. Lu, H. Hofmann, and C. Mi, "A loosely coupled capacitive power transfer system with LC compensation circuit topology," Proc. IEEE Energy Conversion Congress and Exposition, Milwaukee, USA, pp. 1-5, Sep. 2016. DOI: 10.1109/ECCE.2016.7854702

[3] T. Ohira, "Extended $k-Q$ product formulas for capacitive- and inductive-coupling wireless power transfer schemes," IEICE Electron. Express, vol. 11, no. 9, pp. 17, April 2014. DOI: 10.1587/elex.11.20140147

[4] M. Tamura, Y. Naka, K. Murai, and T. Nakata, "Design of a capacitive wireless power transfer system for operation in fresh water," IEEE Trans. Microw. Theory Techn., vol. 62, no. 12, pp. 5873-5884, Dec. 2018. DOI: 10.1109/TMTT.2018. 2875960 
[5] Y. Naka, K. Yamamoto, T. Nakata, and M. Tamura, "Improvement in efficiency of underwater wireless power transfer with electric coupling," IEICE Trans. Electron., vol. E100-C, no. 10, pp. 850-857, Oct. 2017. DOI: 10.1587/transele.E100. C. 850

[6] M. Tamura, Y. Naka, and K. Murai, "Design of capacitive coupler in underwater wireless power transfer focusing on kQ product," IEICE Trans. Electron., vol. E101-C, no. 10, pp. 759-766, Oct. 2018. DOI: 10.1587/transele.E101.C.759

[7] I. Awai, K. Hori, S. Yakuno, and K. Namikoshi, "Wireless power transmission based on directional coupler and directional fileter," Proc. 2010 IEEE MTT-S International Microwave Symposium, Anaheim, CA, USA, pp. 1568-1571, May 2010. DOI: 10.1109/MWSYM.2010.5518186

[8] D.M. Pozar, Microwave Engineering, 4th ed., John Wiley \& Sons, New York, 2011.

\section{Introduction}

Many studies on capacitive wireless power transfer (WPT) use the lumped-constant equivalent circuit $[1,2,3,4,5,6]$. The transfer efficiency shows significant change with frequency in capacitive WPT via water. The change in the transfer efficiency is represented by adding parasitic elements into a conventional equivalent circuit $[4,5,6]$, and is predicted by a voltage input/output ratio derived from the equivalent circuit. However, a relationship between conditions for generating minimum/maximum values of the transfer efficiency and structural parameters of the electrodes in the coupler is not clarified. In this study, an equivalent circuit is proposed via fresh water using the distributed-constant circuit. It is elucidated from our circuit that the minimum and maximum values of the efficiency can be predicted depending on wavelength of the transfer frequency. First, the frequency characteristic of the maximum available efficiency was calculated by electromagnetic (EM) simulations. The lumped-constant equivalent circuit with the parasitic elements represents the characteristic. One study has used a general directional coupler for WPT [7]. We converted the equivalent circuit into the two open-ended directional couplers (i.e., a distributed-constant circuit). Using the couplers, the conditions for generating the minimum and maximum values of the voltage input/output ratio were derived. The efficiencies calculated by the distributed-constant circuit and EM simulation were in good agreement. Finally, it was shown that the minimum and maximum values of the transfer efficiency can be predicted from the structural parameters of the coupler electrodes.

\section{Representation by lumped-constant circuit}

Figures 1(a) and (b) show the coupler model and the equivalent circuit of the capacitive WPT, where \#1-\#3 and \#2-\#4 show the primary and secondary sides, respectively. The capacitances that occurred in \#1-\#4 and \#2-\#3 cannot be ignored, especially when there is misalignment between the primary and secondary sides [2]. The capacitances were ignored because this study assumes that there is perfect symmetry without misalignment. The capacitance $C_{\mathrm{S}}$ and loss $R_{\mathrm{S}}$ occurred at that adjacent electrodes (\#1-\#3,\#2-\#4). Furthermore, $C_{\mathrm{m}}$ and $R_{\mathrm{m}}$ occurred at the facing 


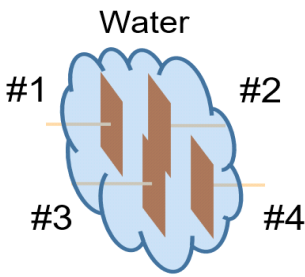

(a) Capacitive couplervia water.

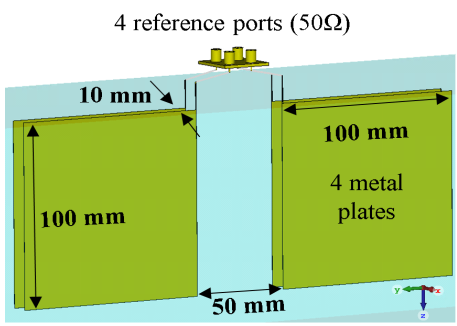

(c) EM simulation model.

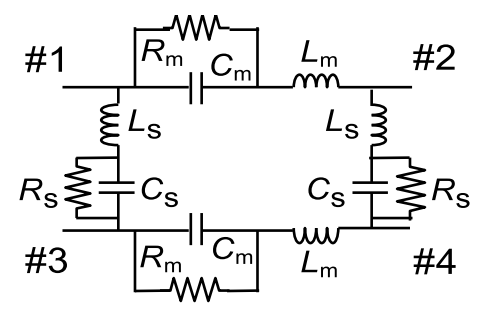

(b) Symmetrical equivalent circuit.

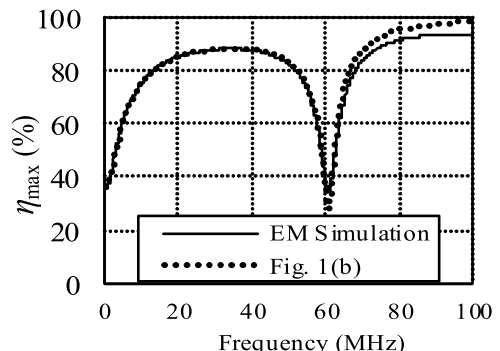

(d) Calculated $\eta_{\max }$ values of (b) and (c).

Fig. 1. Representation by lumped-constant circuit.

electrodes (\#1-\#2,\#3-\#4). $L_{\mathrm{m}}$ and $L_{\mathrm{s}}$ are parasitic inductances in each capacitance. As shown in Fig. 1(c), the coupler model comprises four metal plates and four 50- $\Omega$ reference ports imitated subminiature connectors. Here, tap water is represented using the relative permittivity $\epsilon_{\mathrm{r}}=79$ and the conductivity $\sigma=0.011 \mathrm{~S} / \mathrm{m}$. We calculated the maximum available efficiency $\eta_{\max }$ [3], which indicates the achievable power transfer efficiency under conjugate matching conditions. First, we calculated the four-port single-ended S-parameters using the equivalent circuit (advanced design system) and EM simulation (CST microwave studio) and converted them into two-port differential S-parameters. Next, the results were converted into the twoport impedance matrix. Finally, $\eta_{\max }$ was calculated by substituting the impedance results into the following two equations:

$$
\begin{gathered}
\alpha=\frac{\left|Z_{21}\right|}{\sqrt{R_{11} R_{22}-R_{12} R_{21}}} . \\
\eta_{\max }=1-\frac{2}{1+\sqrt{1+\alpha^{2}}} .
\end{gathered}
$$

Figure 1(d) shows the calculated $\eta_{\max }$ values. The element values are as follows: $C_{\mathrm{s}}=72 \mathrm{pF}, R_{\mathrm{s}}=960 \Omega, L_{\mathrm{s}}=95 \mathrm{nH}, C_{\mathrm{m}}=840 \mathrm{pF}, R_{\mathrm{m}}=74 \Omega$, and $L_{\mathrm{m}}=8.5 \mathrm{nH}$. The $\eta_{\max }$ values are calculated from the equivalent circuit in Fig. 1(b) and EM simulation, which are in good agreement up to around $60 \mathrm{MHz}$. However, the relationship between the conditions for generating the minimum/maximum values of the transfer efficiency and the structural parameters of the electrodes in the coupler is not clarified.

\section{Representation by distributed-constant circuit}

\subsection{Formulation of the voltage input/output ratio}

Figure 2 shows the procedure of the conversion from the capacitive coupler into the directional coupler. To simplify the figures, $L_{\mathrm{s}}, L_{\mathrm{m}}, R_{\mathrm{s}}$ and $R_{\mathrm{m}}$ are not listed. Figure 2(a) is represented as the balanced circuit topology by including the power 


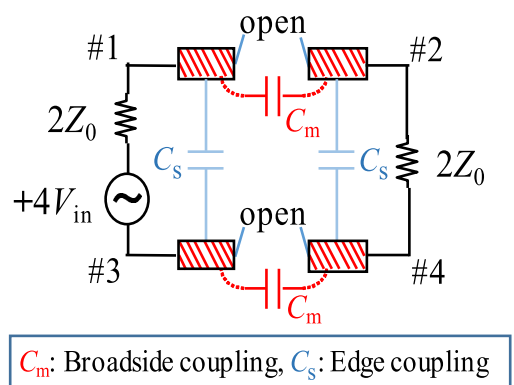

(a) Balanced circuit topology of Fig.1(b).

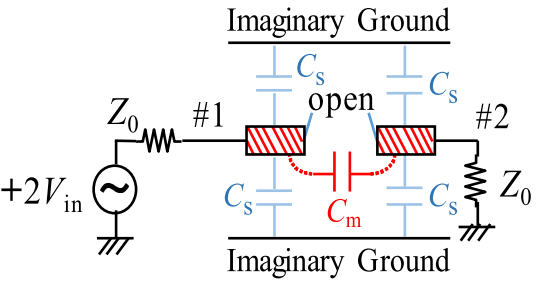

(c) Unbalanced representation (\#1 to \#2).

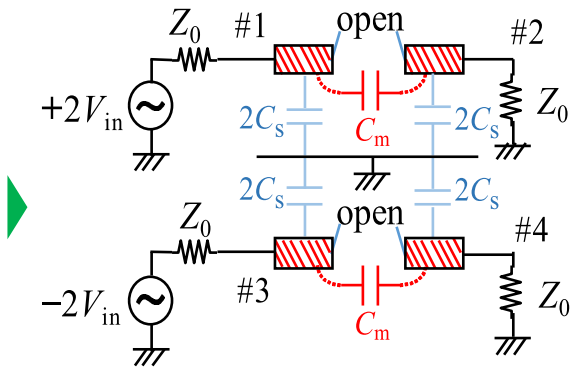

(b) Conversion by using imaginary ground.

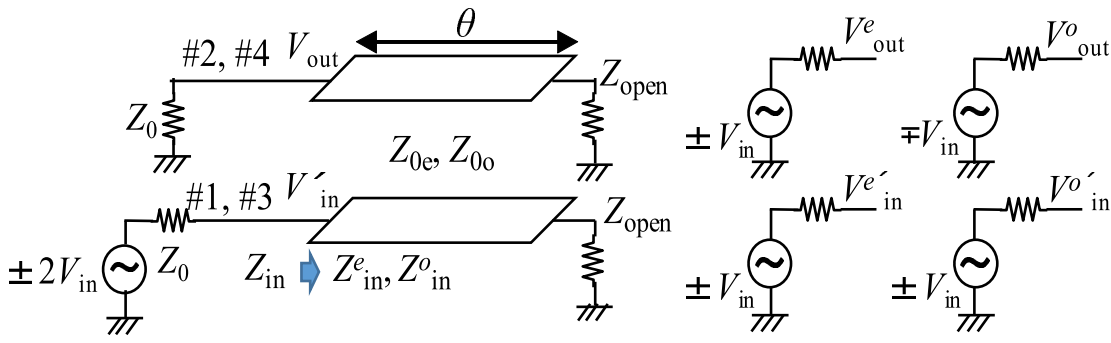

(e) Conversion from (c) and (d) into directional coupler.

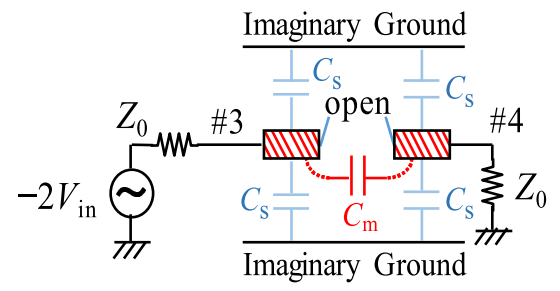

(d) Unbalanced representation (\#3 to \#4). 
lines for the odd mode in Fig. 2(g). The capacitances of the two modes are expressed by Eqs. (3) and (4) [8]. Equations (5) and (6) show the characteristic impedances for the even and odd modes. Note that $R_{\mathrm{e}}$ and $R_{\mathrm{o}}$ show the loss generated at the inductances $L_{\mathrm{e}}$ and $L_{\mathrm{o}}$, respectively, in each mode. Furthermore, $G_{\mathrm{e}}$ and $G_{\mathrm{o}}$ show the loss generated in Eqs. (3) and (4). Based on the results of Section 2, the conditions $R_{\mathrm{s}}>1 /\left(\omega C_{\mathrm{s}}\right)$ and $R_{\mathrm{m}}>1 /\left(\omega C_{\mathrm{m}}\right)$ are satisfied at $3 \mathrm{MHz}$. Therefore, we apply the lossless transmission line theory for conversion into the directional coupler as follows:

$$
\begin{aligned}
& C_{\mathrm{e}}=C_{\mathrm{in}-\mathrm{GND}}=C_{\mathrm{out}-\mathrm{GND}} . \\
& C_{\mathrm{o}}=C_{\mathrm{in}-\mathrm{GND}}+2 C_{\mathrm{in}-\mathrm{out}}=C_{\mathrm{out}-\mathrm{GND}}+2 C_{\text {in-out }} . \\
& Z_{0 \mathrm{e}}=\sqrt{\frac{R_{\mathrm{e}}+j \omega L_{\mathrm{e}}}{G_{\mathrm{e}}+j \omega C_{\mathrm{e}}}} \approx \sqrt{\frac{L_{\mathrm{e}}}{C_{\mathrm{e}}}} . \\
& Z_{0 \mathrm{o}}=\sqrt{\frac{R_{\mathrm{o}}+j \omega L_{\mathrm{o}}}{G_{\mathrm{o}}+j \omega C_{\mathrm{o}}}} \approx \sqrt{\frac{L_{\mathrm{o}}}{C_{\mathrm{o}}}} .
\end{aligned}
$$

The input impedance for each mode is expressed by Eqs. (7) and (8). These equations can be simplified because of the open condition of $Z_{\text {open }}$.

$$
\begin{aligned}
& Z_{\text {in }}^{e}=Z_{0 \mathrm{e}} \frac{Z_{\text {open }}+j Z_{0 \mathrm{e}} \tan \theta}{Z_{0 \mathrm{e}}+j Z_{\text {open }} \tan \theta} \approx \frac{Z_{0 \mathrm{e}}}{j \tan \theta} . \\
& Z_{\text {in }}^{o}=Z_{0 \mathrm{o}} \frac{Z_{\text {open }}+j Z_{0 \mathrm{o}} \tan \theta}{Z_{0 \mathrm{o}}+j Z_{\text {open }} \tan \theta} \approx \frac{Z_{0 \mathrm{o}}}{j \tan \theta} .
\end{aligned}
$$

The voltages at \#1 for each mode are listed below:

$$
V_{\mathrm{in}}^{e \prime}=V_{\mathrm{in}}^{\prime} \frac{Z_{\mathrm{in}}^{e}}{Z_{\mathrm{in}}^{e}+Z_{0}}, \quad V_{\mathrm{in}}^{o \prime}=V_{\mathrm{in}}^{\prime} \frac{Z_{\mathrm{in}}^{o}}{Z_{\mathrm{in}}^{o}+Z_{0}} .
$$

Here, as long as $Z_{\text {in }}=Z_{0}$ is satisfied, we have $V_{\text {in }}^{\prime}=V_{\text {in }}$ by voltage division in Fig. 2(e) because there was no voltage reflection. Moreover, by the preceding voltage definition in Fig. 2(f), $V_{\text {out }}$ is expressed as follows:

$$
V_{\text {out }}=V_{\text {out }}^{e}+V_{\text {out }}^{o}=V_{\text {in }}^{e \prime}+\left(-V_{\text {in }}^{o \prime}\right)=V_{\text {in }}\left[\frac{Z_{\text {in }}^{e}}{Z_{\text {in }}^{e}+Z_{0}}-\frac{Z_{\text {in }}^{o}}{Z_{\text {in }}^{o}+Z_{0}}\right] .
$$

Equation (10) reduces to Eq. (11) using Eqs. (7) and (8). The voltage ratio of $V_{\text {out }} / V_{\text {in }}$ is given by Eq. (12).

$$
\begin{gathered}
V_{\text {out }}=V_{\text {in }}\left[\frac{Z_{0 \mathrm{e}}}{Z_{0 \mathrm{e}}+j Z_{0} \tan \theta}-\frac{Z_{0 \mathrm{o}}}{Z_{0 \mathrm{o}}+j Z_{0} \tan \theta}\right] . \\
\frac{V_{\text {out }}}{V_{\text {in }}}=\frac{Z_{0 \mathrm{e}}-Z_{0 \mathrm{o}}}{Z_{0 \mathrm{e}}+Z_{0 \mathrm{o}}+j\left[Z_{0} \tan \theta-Z_{0 \mathrm{e}} Z_{0 \mathrm{o}} /\left(Z_{0} \tan \theta\right)\right]} .
\end{gathered}
$$

From Eq. (12), we confirmed that the minimum and maximum values of the voltage ratio occurred when the imaginary parts were equal to $\pm \infty$ and 0 , respectively. Furthermore, $\theta_{\min }$ in Eq. (13) shows the phase difference when the imaginary part was equal to $\pm \infty$; the minimum values occurred when the phase difference was $(n-1) \pi / 2$ (with the wavelengths $0, \lambda / 4$, and so on). From Eq. (14), $\arctan \pm 1$ (the longest wavelength is $\lambda / 8$ ) holds when $\sqrt{Z_{0 \mathrm{e}} Z_{0 \mathrm{o}}}=Z_{0}$ is satisfied. If all the 
ports were terminated at $Z_{0}$ in Fig. 2(e), we would have $\sqrt{Z_{0 \mathrm{e}} Z_{0 \mathrm{o}}}=Z_{0}$ [8]. However, $\arctan \pm 1(\lambda / 8)$ does not hold because $Z_{\text {open }} \neq Z_{0}$. Therefore, the wavelength having the maximum values are determined by the $\sqrt{Z_{0 \mathrm{e}} Z_{0 \mathrm{o}}} / Z_{0}$ ratio.

$$
\begin{gathered}
\theta_{\min }=\arctan 0, \arctan ( \pm \infty)=\frac{(n-1) \pi}{2}(n: 1,2,3, \cdots) . \\
\theta_{\max }=\arctan \left( \pm \frac{\sqrt{Z_{0 \mathrm{e}} Z_{0 \mathrm{o}}}}{Z_{0}}\right) .
\end{gathered}
$$

\subsection{Verification}

Figure 3(a) shows the equivalent circuit represented by two open-ended directional couplers. For verification, we assign concrete values to $\epsilon_{\mathrm{r}}, \sigma, S, L, W$, and $B$ in Fig. $2(\mathrm{~g})$. Here, $L$ is the transmission length across which the phase difference occurs. The phase difference is governed by the length of the electrode, which is affected by the wavelength shorting derived from $\epsilon_{\mathrm{r}}$. Therefore, $L$ is the diagonal length of the electrode $(141 \mathrm{~mm})$ in Fig. $1(\mathrm{c}) . W$ is $100 \times 100 / 141=71 \mathrm{~mm}$, which ensures that the area size is constant. Based on the information in Section 2, we specify $\epsilon_{\mathrm{r}}=79, \sigma=0.011 \mathrm{~S} / \mathrm{m}$, and the distance between the facing electrodes $S=10 \mathrm{~mm}$. $C_{\mathrm{s}}$ corresponds to $C_{\mathrm{in}-\mathrm{GND}}$ and $C_{\text {out-GND }}$, as shown in Fig. $2(\mathrm{~g}) . C_{\mathrm{s}}$ is the edge-coupled capacitance, and the distance is $50 \mathrm{~mm}$. However, $C_{\mathrm{in}-\mathrm{GND}}$ and $C_{\text {out-GND }}$ are the broadside-coupled capacitances and the distance is $(B-S) / 2$. We compared the capacitances in the broadside-coupled and edge-coupled states, and specified that the broadside $>$ edge. Therefore, $B$ was more than $50 \mathrm{~mm}$ in length, and the fitting result was $360 \mathrm{~mm}$.

Figure 3(b) shows the calculated $\eta_{\max }$ values; the minimum value was $59.8 \mathrm{MHz}$, and maximum values was $35.1 \mathrm{MHz}$. When we considered the effect of the wavelength shorting, we reported that $59.8 \mathrm{MHz}$ was $\lambda / 4$, and $29.9 \mathrm{MHz}$ was $\lambda / 8$ of $L=141 \mathrm{~mm}$. The minimum values were in good agreement. The calculated results of Eq. (12) with $Z_{0}=1, Z_{0 \mathrm{e}}=4$ and the various $Z_{0 \mathrm{o}}$ values of $0.125,0.25$, and 0.5 are shown in Fig. 3(c). $\sqrt{Z_{0 \mathrm{e}} Z_{0 \mathrm{o}}}=Z_{0}$ holds at $Z_{0 \mathrm{o}}=0.25$, and the maximum value occurred in $\lambda / 8\left(45^{\circ}\right)$. Moreover, we confirmed that the center around $\lambda / 8$ was affected by the change in the $\sqrt{Z_{0 \mathrm{e}} Z_{0 \mathrm{o}}} / Z_{0}$ ratio. From the above, we observe that the difference between $29.9 \mathrm{MHz}$ and $35.1 \mathrm{MHz}$ occurred because $\sqrt{Z_{0 \mathrm{e}} Z_{0 \mathrm{o}}}>Z_{0}$.

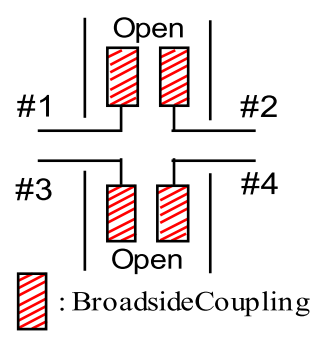

(a) Representation by two openended directional couplers.

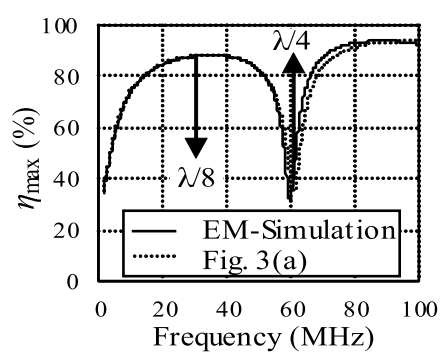

(b) Comparison $\eta_{\max }$ between EM simulation and (a).

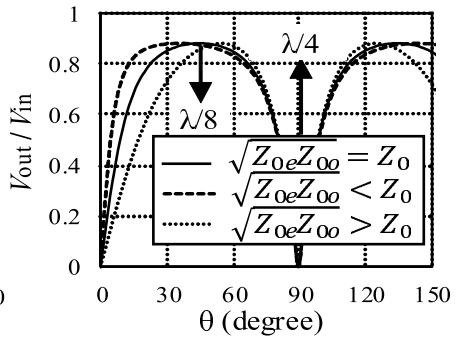

(c) Result of Eq. (12) with various $\sqrt{Z_{0 e^{Z_{0 o}}}}$.

Fig. 3. Representation of distributed-constant circuits. 


\section{Conclusion}

In this study, we represented the equivalent circuit of the capacitive WPT using a distributed-constant circuit. The lumped-constant equivalent circuit was converted into the two open-ended directional couplers. Consequently, we determined the condition for the generation of minimum and maximum value of the voltage ratio. The $\eta_{\max }$ values were calculated using the distributed-constant equivalent circuit and EM simulation, which were in good agreement. Finally, we clarified that the minimum and maximum $\eta_{\max }$ values occurred at 0 and $\lambda / 4$, respectively, and centered around $\lambda / 8$. The changes in $\lambda / 8$ depended on the $\sqrt{Z_{0 \mathrm{e}} Z_{0 \mathrm{o}}} / Z_{0}$ ratio.

\section{Acknowledgments}

This work was supported in part by the VLSI Design and Education Center, The University of Tokyo in collaboration with Agilent Technologies Japan, Ltd., and in part by JSPS KAKENHI under Grant JP18K04262. 